\title{
Adaptive beam shaping by controlled thermal lensing in optical elements
}

\author{
Muzammil A. Arain, Volker Quetschke, Joseph Gleason, Luke F. Williams, Malik Rakhmanov, \\ Jinho Lee, Rachel J. Cruz, Guido Mueller, D. B. Tanner, and David. H. Reitze
}

\begin{abstract}
We describe an adaptive optical system for use as a tunable focusing element. The system provides adaptive beam shaping via controlled thermal lensing in the optical elements. The system is agile, remotely controllable, touch free, and vacuum compatible; it offers a wide dynamic range, aberration-free focal length tuning, and can provide both positive and negative lensing effects. Focusing is obtained through dynamic heating of an optical element by an external pump beam. The system is especially suitable for use in interferometric gravitational wave interferometers employing high laser power, allowing for in situ control of the laser modal properties and compensation for thermal lensing of the primary laser. Using $\mathrm{CO}_{2}$ laser heating of fused-silica substrates, we demonstrate a focal length variable from infinity to $4.0 \mathrm{~m}$, with a slope of 0.082 diopter/W of absorbed heat. For on-axis operation, no higher-order modes are introduced by the adaptive optical element. Theoretical modeling of the induced optical path change and predicted thermal lens agrees well with measurement. @ 2007 Optical Society of America

OCIS codes: $\quad 20.6810,010.1080,350.5830$.
\end{abstract}

\section{Introduction}

When an optical element is subjected to nonuniform spatial heat distribution (as even a weakly absorbing element is when a high-power Gaussian laser beam passes through it), its physical surface profile and refractive index change. The nonuniform heat distribution can arise from heating with an optical beam incident on the optical element or from some external heat source. The absorbed power creates a nonuniform temperature distribution in the element, causing both shape deformations and spatially dependent refractive indices to appear. The dominant effects are surface deformation, governed by the coefficient of linear expansion $\left(\alpha_{T}\right)$, and changes in refractive index, depending upon the thermo-optic coefficient $(\mathrm{d} n / \mathrm{d} T)$. For most optical materials, other effects, the thermoelastic coefficient, and the photoelastic effect are smaller and therefore are neglected. ${ }^{1}$ However, for materials with large thermal expansion coeffi-

The authors are with the Department of Physics, University of Florida, Gainesville, Florida 32611, USA. M. A. Arain's e-mail address is muzamil@phys.ufl.edu.

Received 23 August 2006; accepted 24 November 2006; posted 4 December 2006 (Doc. ID 74353); published 3 April 2007.

0003-6935/07/122153-13\$15.00/0

(C) 2007 Optical Society of America cient, this effect should be included. The dominant effects may create substantial thermal aberrations, i.e., position-based optical path-length change, in the optical beam. This behavior is typically modeled by introducing imaginary lenses at the surface and in the substrate. Hence, this effect is termed "thermal lensing." The focal length [or the radius of curvature (ROC)] of this lens varies according to the geometry and physical properties of the material, the temperature profile, and the incident optical beam.

The thermal lens can be used to realize adaptive optical components provided that the heating of the element is precisely controlled. ${ }^{2,3}$ The ROCs of these elements can be changed by heating them with a pump beam or heating beam in conjunction with the probe or original beam. (In this paper, we use the term "probe beam" to refer to the beam that suffers the thermal lens on account of its own absorption. We use interchangeably "pump beam" or "heating beam" to refer to the beam used to compensate the thermal lensing and to control the profile of the probe beam.) By controlling the intensity and the beam diameter of the heating beam, the optical element can be transformed into a powerful modal shaping system, correcting in situ errors due to undesired changes in the optical systems as well as serving as a fine-focusing system. Recently such an adaptive system was proposed, and initial results were presented. ${ }^{4}$ Here we 
present a comprehensive investigation of thermally induced adaptive beam shaping, including detailed theory and enhanced experimental results. A number of new techniques is proposed to implement the positive as well as negative lensing element. Issues such as the correct ROC estimation, higher-order losses, response time, and beam deviations are studied both experimentally and theoretically.

\section{Theoretical Foundations}

We begin by calculating the three-dimensional (3D) temperature distributions in optical elements. While a number of techniques are available to model this behavior, the basis of all these methods is the thermal diffusion equation. This equation can be solved by both analytical and numerical finite-elementanalysis methods. ${ }^{5-8}$ Some simpler methods based upon geometrical considerations also exist but can be quite inaccurate for high-power applications..$^{9,10}$

Hello and Vinet ${ }^{5,6}$ provided an analytical solution for the thermal aberrations in a finite cylinder due to absorption from an optical beam. The model is general and can be applied to a large number of situations; it is particularly suitable for thermal lensing calculations in large-scale gravitational wave interferometers. One specific limitation is in the case of nonuniform absorption or point absorption, where finite-element-analysis methods become necessary.

We use a MATLAB-based implementation of the Hello and Vinet model ${ }^{11}$ as applicable to the geometry of laser gravitational wave interferometers, shown in Fig. 1. Light is incident on a cylindrical optical element of thickness $h$ and radius $a$ with an optical coating on the surfaces. The optical element is assumed to be in vacuum where the only heat escape mechanism is through thermal radiation. The front surface has an antireflection coating at the probe beam wavelength while the back surface has a high reflective coating. The optical beam has power $P$ and wavelength $\lambda_{0}$. The element has coating (or surface) absorption $a_{c}$ and

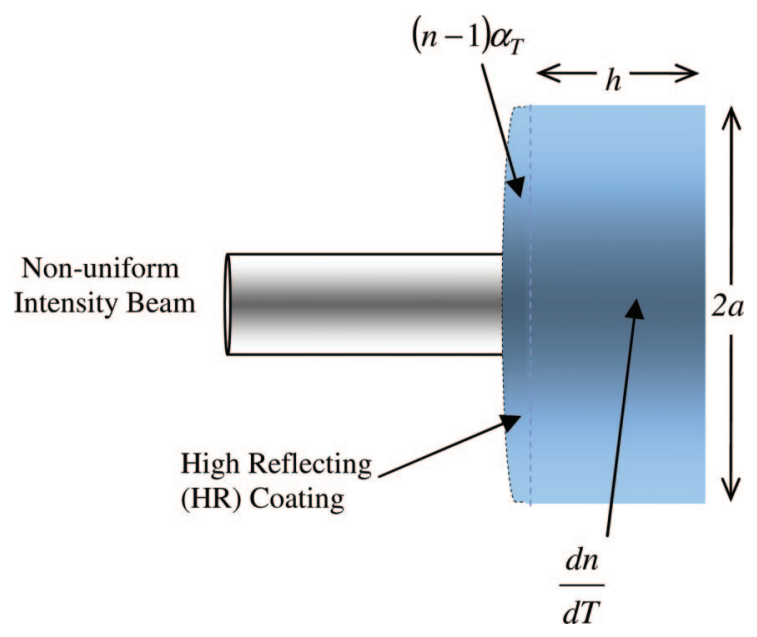

Fig. 1. (Color online) Non-uniform-intensity beam incident on an optical element. The power absorbed in the coating or the surface creates thermal aberrations on the surface due to the thermal expansion coefficient $\alpha_{T}$ and due to the $\mathrm{d} n / \mathrm{d} T$ in the substrate. substrate (or bulk) absorption coefficient $\alpha_{s}$ (in $\mathrm{m}^{-1}$ ), refractive index $n$, thermo-optic coefficient $\mathrm{d} n / \mathrm{d} T\left(\mathrm{~K}^{-1}\right)$, thermal conductivity $\kappa_{T}\left(\mathrm{~W} \mathrm{~m}^{-1} \mathrm{~K}^{-1}\right)$, total emissivity $\varepsilon$, and Poisson ratio $\nu$. The temperature distribution $T_{s}$ due to substrate heating throughout the material and temperature distribution $T_{c}$ due to surface heating is given by ${ }^{11}$

$$
T_{s}(r, z)=P \frac{\alpha_{s} a^{2}}{k_{t}} \sum_{k} \frac{p_{k}}{\zeta_{k}^{2}} \times\left[1-2 \tau A_{k} \cosh \left(\zeta_{k} \frac{z}{a}\right)\right] J_{0}\left(\zeta_{k} \frac{r}{a}\right),
$$

$$
\begin{aligned}
T_{c}(r, z)= & P \frac{a_{c} a}{k_{t}} \sum_{k} p_{k} \times\left[A_{k} \cosh \left(\zeta_{k} \frac{z}{a}\right)-B_{k} \sinh \left(\zeta_{k} \frac{z}{a}\right)\right] \\
& \times J_{0}\left(\zeta_{k} \frac{r}{a}\right),
\end{aligned}
$$

where $\tau \equiv 4 \varepsilon \sigma T_{\text {ext }}^{3}\left(a / \kappa_{t}\right)$ with $\sigma$ as the StefanBoltzmann constant. The coefficients $A_{k}$ and $B_{k}$ are given by

$$
\begin{aligned}
& A_{k}=\frac{1}{2\left[\zeta_{k} \sinh \left(\gamma_{k}\right)+\tau \cosh \left(\gamma_{k}\right)\right]}, \\
& B_{k}=\frac{1}{2\left[\zeta_{k} \cosh \left(\gamma_{k}\right)+\tau \sinh \left(\gamma_{k}\right)\right]},
\end{aligned}
$$

where $\gamma_{k} \equiv \zeta_{k}(h / 2 a)$. The terms in these series are characterized by the roots of the equation

$$
\zeta J_{1}(\zeta)-\tau J_{0}(\zeta)=0
$$

To increase the accuracy of our calculations, numerical techniques are used to find the exact roots of the equation instead of using the approximation $\zeta_{k} \cong(k$ $+1 / 4) \pi$ for $k \in\{0,1,2, \ldots\}$ as done in Ref. 11. The constants $p_{k}$ are the coefficients of a Dini series ${ }^{5}$ expansion of the incident intensity distribution $I(r)$. These coefficients can be calculated numerically

$$
p_{k}=\frac{2 \zeta_{k}^{2}}{\left(\zeta_{k}^{2}+\tau^{2}\right) J_{0}^{2}\left(\zeta_{k}\right)} \frac{1}{a^{2}} \int_{0}^{a} r J_{0}\left(\zeta_{k} \frac{r}{a}\right) I(r) \mathrm{d} r
$$

For example, a normalized $\mathrm{TEM}_{00}$ Gaussian beam, a "top-hat" beam, and their Dini series expansions are given in Fig. 2; good agreement is seen.

Once the temperature distribution is obtained, the substrate thermal aberrations can be calculated by

$$
\phi(r) \equiv \frac{2 \pi}{\lambda_{0}} \frac{\mathrm{d} n}{\mathrm{~d} T} \int_{-h / 2}^{h / 2} T(r, z) \mathrm{d} z
$$

By using the temperature distributions calculated earlier, the contributions of coating and substrate 


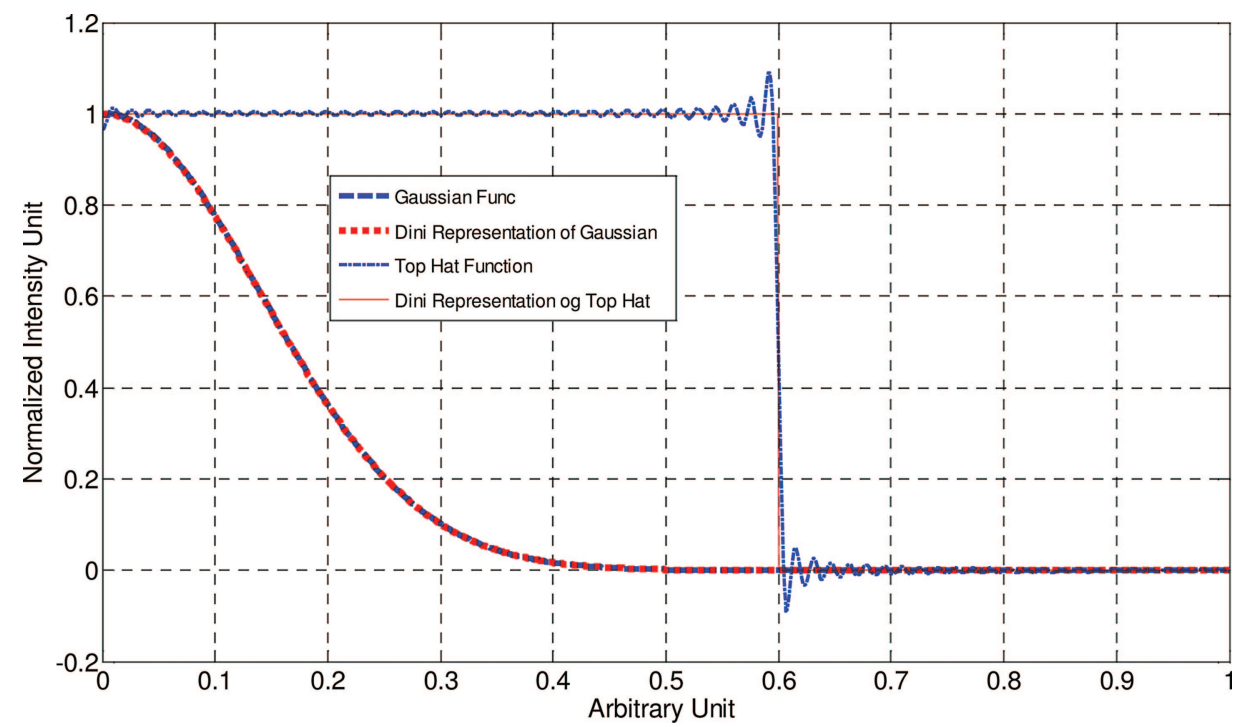

Fig. 2. (Color online) Dini series representation of a top-hat beam and a Gaussian beam using the first 40 terms of the expansion.

heating are

$$
\begin{aligned}
\phi_{s}(r)= & \alpha_{s} h P \frac{2 \pi a^{2}}{\lambda_{0} k_{T}} \frac{\mathrm{d} n}{\mathrm{~d} t} \sum_{k} \frac{P_{k}}{\zeta_{k}^{2}} \times\left[1-\frac{2 \tau A_{k}}{\gamma_{k}} \sinh \left(\gamma_{k}\right)\right] \\
& \times J_{0}\left(\zeta_{k} \frac{r}{a}\right), \\
\phi_{c}(r)= & a_{c} P \frac{2 \pi a^{2}}{\lambda_{0} k_{T}} \frac{\mathrm{d} n}{\mathrm{~d} t} \sum_{k} \frac{P_{k}}{\zeta_{k}} \times 2 A_{k} \sinh \left(\gamma_{k}\right) J_{0}\left(\zeta_{k} \frac{r}{a}\right) .
\end{aligned}
$$

Another effect of this nonuniform distribution is the change in surface profile of the optical element. Here only the axial direction surface change is taken into account. The surface deformation $u_{c}$ due to coating or surface absorption and $u_{s}$ due to substrate absorption is ${ }^{11}$

$$
\begin{aligned}
u_{c}(r, \pm h / 2)= & \pm a_{c} P \frac{\alpha_{T}(1+v) a^{2}}{k_{T}} \sum_{k} \frac{p_{k}}{\zeta_{k}^{2}} \times \frac{\sinh \left(\gamma_{k}\right)}{\gamma_{k}} \\
& \times\left[\frac{\sinh \left(\gamma_{k}\right)}{\gamma_{k}+\sinh \left(\gamma_{k}\right) \cosh \left(\gamma_{k}\right)}-\tau A_{k}\right] \\
& \times\left[J_{0}\left(\zeta_{k} \frac{r}{a}\right)-1\right], \\
u_{s}(r, \pm h / 2)= & \pm \alpha_{s} h P \frac{\alpha_{T}(1+v) a^{2}}{k_{T}} \sum_{k} \frac{p_{k}}{\zeta_{k}^{2}} \\
& \times\left[A_{k} \cosh \left(\gamma_{k}\right) \mp B_{k} \sinh \left(\gamma_{k}\right)\right] \\
& \times\left[J_{0}\left(\zeta_{k} \frac{r}{a}\right)-1\right]-\frac{3}{2} \alpha_{s} h P \\
& \times \frac{\alpha_{T}(1-v) a^{2}}{k_{T}} \sum_{k} \frac{r^{2} p_{k}}{a^{2} \zeta_{k}^{2}} B_{k} \\
& \times\left[\frac{\sinh \left(\gamma_{k}\right)}{\gamma_{k}}-\cosh \left(\gamma_{k}\right)\right] J_{1}\left(\zeta_{k}\right) .
\end{aligned}
$$

The surface deformation given by Eq. (8) combined with the original ROC gives the modified ROC of both sides of the optical element. The solution presented here is a steady-state solution and therefore once a specified amount of heat is deposited, the resultant thermal lens will retain its modal properties as long as the deposited heat distribution and amount remains same.

One notable limitation of this treatment is the use of the linearized radiative boundary condition. The radiative heat loss of an element at temperature $T$ is given by

$$
F=\sigma^{\prime}\left\lfloor T^{4}-T_{e x t}^{4}\right\rfloor
$$

where $T_{\text {ext }}{ }^{4}$ is the ambient temperature and $\sigma^{\prime}$ is the Stefan-Boltzmann constant corrected for emissivity. If the temperature rise $\Delta T$ of the element is not exces-

Table 1. Nominal Values for Advanced LIGO Cavity Mirrors (Test Masses)

\begin{tabular}{lcc}
\hline \multicolumn{1}{c}{ Property } & Unit & Value \\
\hline Diameter & $\mathrm{cm}$ & 34 \\
Thickness & $\mathrm{cm}$ & 30 \\
Material & - & $\mathrm{FS}$ \\
Hot ROC & $\mathrm{m}$ & 2076 \\
Beam size & $\mathrm{cm}$ & 6.0 \\
Power incident on HR side & $\mathrm{kW}$ & 850 \\
Power through substrate & $\mathrm{kW}$ & 2.1 \\
Refractive index at $1 \mu \mathrm{m}$ & - & 1.45 \\
Bulk absorption at $1 \mu \mathrm{m}$ & parts per $10^{6} \mathrm{~cm}^{-1}$ & 2 \\
Thermo-optic coefficient & $10^{-6} \mathrm{~K}^{-1}$ & 8.7 \\
Thermal conductivity & $\mathrm{W} \mathrm{m}^{-1} \mathrm{~K}^{-1}$ & 1.37 \\
Heat capacity & $\mathrm{J} \mathrm{kg}^{-1} \mathrm{~K}^{-1}$ & 739 \\
Thermal expansion & $10^{-6} \mathrm{~K}^{-1}$ & 0.55 \\
Density & $\mathrm{Kg} \mathrm{m}^{-3}$ & 22,010 \\
Poisson ratio & - & 0.17 \\
\hline
\end{tabular}


sive, usually the case, Eq. (9) can be approximated as

$$
F=4 \sigma^{\prime} T_{e x t}{ }^{3} \Delta T \text {. }
$$

An ideal spherical lens has a quadratic variation of physical path length with radius; an ideal gradedindex lens has a refractive index, which varies quadratically with radius. In contrast to this, the thermal aberrations may not be exactly quadratic. To pick a concrete example, we first model the expected thermal lensing in the proposed advanced LIGO interferometer, specifically the arm-cavity input test mass. ${ }^{12}$ Table 1 gives the mirror and cavity parameters while Fig. 3 shows the substrate thermal lens for a typical coating absorbed power of $0.5 \mathrm{~W}$. It is evident that the induced substrate aberrations, plotted as a solid curve, are not quadratic. Therefore, an approximation is required to include the thermal lens in the cavity design. For homogeneous absorption, the surface deformation or the thermal aberrations inside the substrate can be represented by a higher-order polynomial:

$$
s(x)=\sum_{m=0}^{M} A_{m} x^{m}
$$

Instead of applying geometrical approximations or using the coefficient of the quadratic term in Eq. (11) as a representative thermal lens, ${ }^{10}$ a general method that can be applied to estimate the ROC in thermal lensing due to both surface deformation and heating in the substrate is presented.

Consider an optical beam with a fundamental Gaussian TEM $_{00}$ profile incident on an optical surface of ROC $R_{1}$ with a minimum beam waist of $w_{0}$ at $z=0$, beam waist $w(z)$ at a distance $z$ from the origin, and phase front radius of curvature $R(z)$ as shown in Fig. 4. The incident electric field in the fundamental Gaussian beam is given by

$$
E_{1}(x, z)=\left(\frac{2}{\pi}\right)^{1 / 4} \frac{1}{\sqrt{w(z)}} \exp -x^{2}\left[\frac{1}{w^{2}(z)}+i \frac{\pi}{\lambda R(z)}\right]
$$

The actual reflected and transmitted beam from the surface is given by

$$
\begin{aligned}
E_{2}(x, z)= & \left(\frac{2}{\pi}\right)^{1 / 4} \frac{1}{\sqrt{w(z)}} \exp -x^{2}\left[\frac{1}{w^{2}(z)}+i \frac{\pi}{\lambda R(z)}\right. \\
& \left.-i \frac{2 \pi}{\lambda R_{1}}+i \frac{\pi}{\lambda} \frac{4 s(x)}{x^{2}}\right] .
\end{aligned}
$$

Here $\left(\pi / \lambda_{1}\right)\left[4 s(x) / x^{2}\right]$ is the optical phase difference introduced by the actual thermal lens. In case of reflection, this term represents the surface deformations while in the case of transmission, this term represents the sum of surface and substrate aberrations. Now, the electric field $E_{3}(x, z)$ reflected and transmitted by the imaginary perfect thermal lens

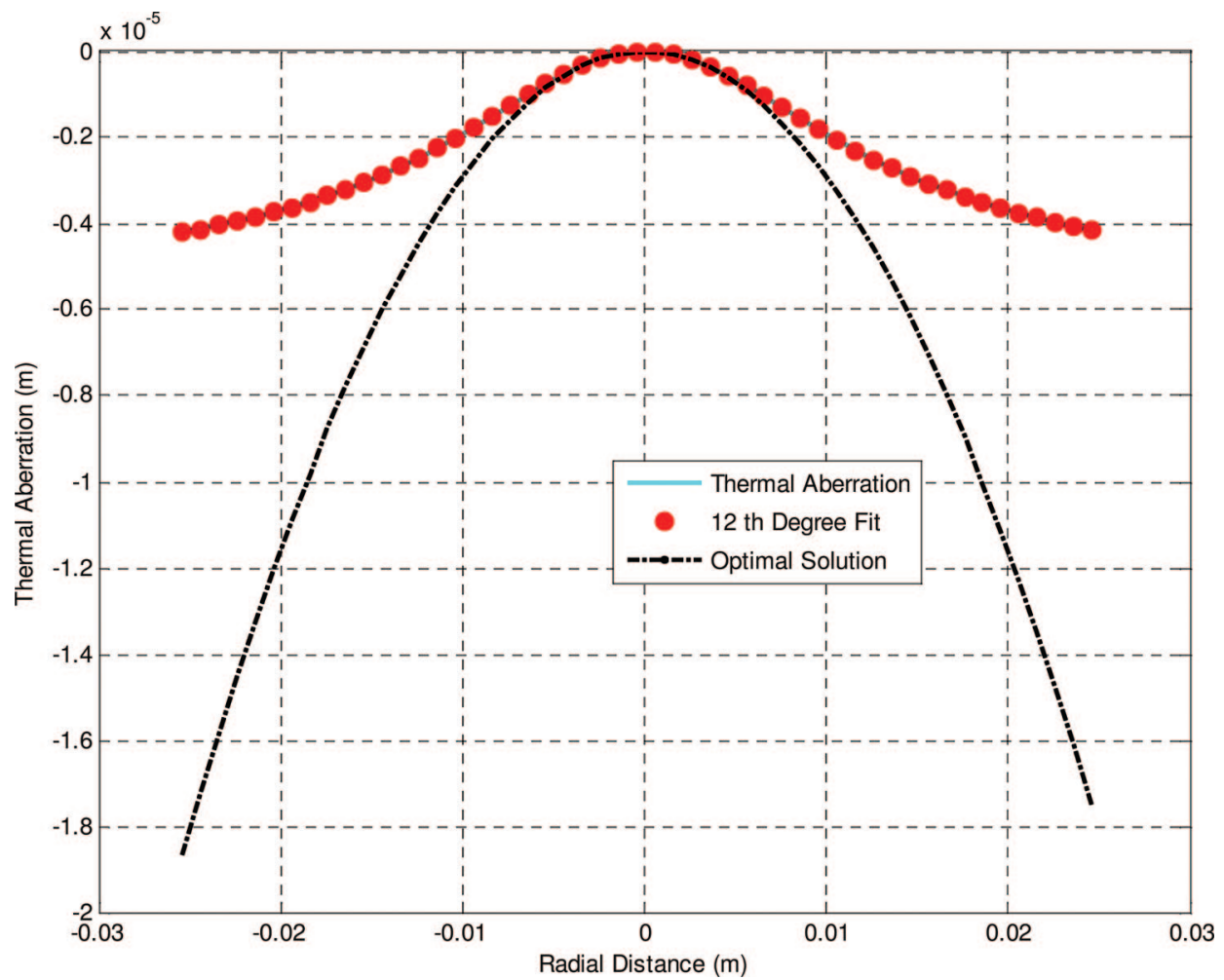

Fig. 3. (Color online) Thermal aberration in the substrate mirror due to $0.5 \mathrm{ppm}$ absorption in the coating for the case of Table 1 data plotted as a solid curve. The approximation using the 12th degree polynomial is plotted as circles, and the optimal solution is plotted as a dotted-dashed curves. 

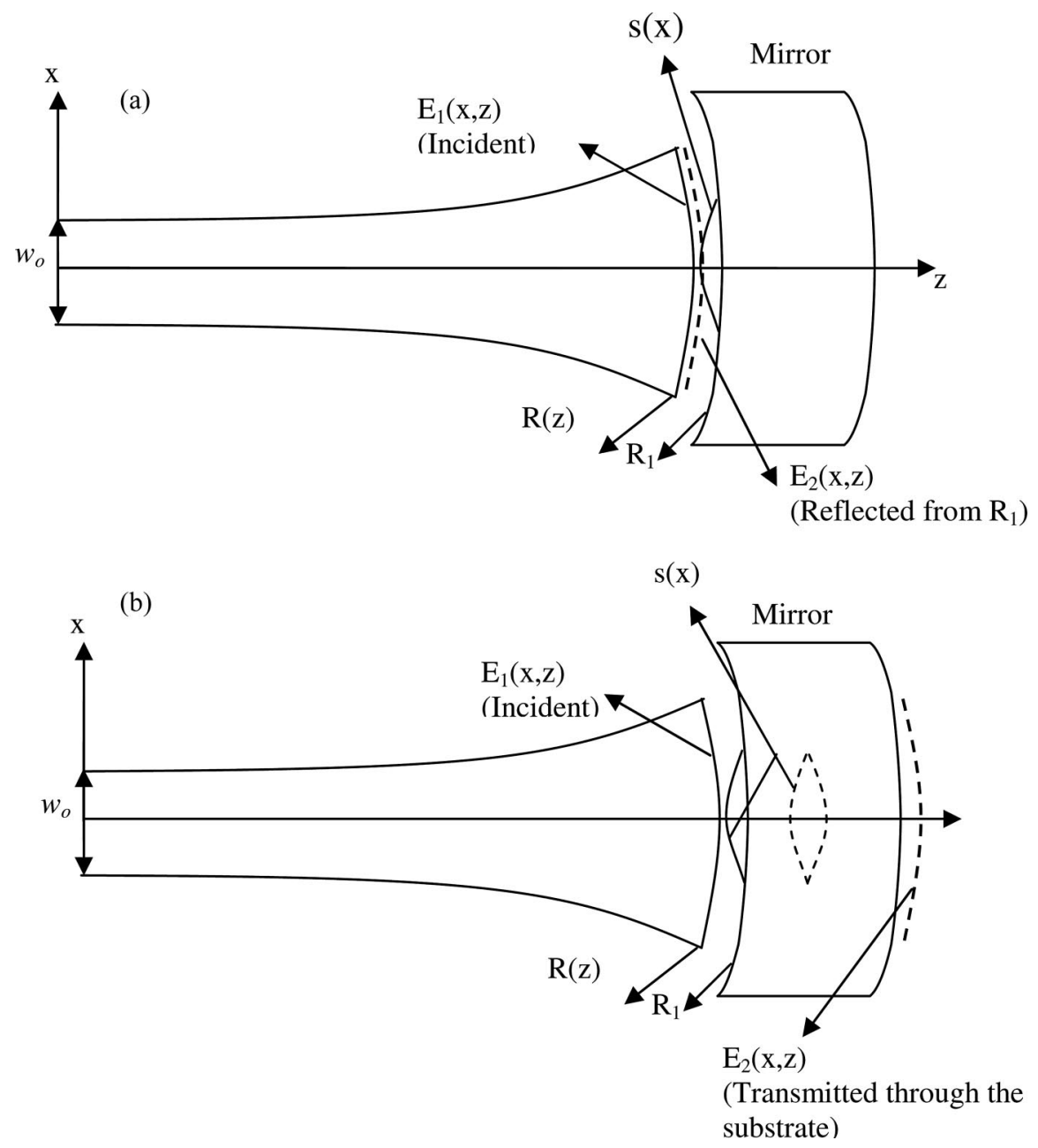

Fig. 4. Geometry of the incident, reflected, and transmitted electric fields from an optical material with thermal lensing. In case of reflection, the thermal aberrations consist of surface deformation as shown in (a) while in transmission, thermal deformations represent the sum of surface and substrate aberrations as depicted in (b).

with ROC of $R_{\text {opt }}$ can be expressed as

$$
\begin{aligned}
E_{3}(x, z)= & \left(\frac{2}{\pi}\right)^{1 / 4} \frac{1}{\sqrt{w(z)}} \exp -x^{2}\left[\frac{1}{w^{2}(z)}+i \frac{\pi}{\lambda R(z)}\right. \\
& \left.-i \frac{2 \pi}{\lambda R_{1}}+i \frac{\pi}{\lambda} 4 A_{\text {opt }}\right]
\end{aligned}
$$

where $A_{o p t}$ is the optimized value of the coefficient used to represent the thermal lens and is equal to $A_{\text {opt }}=1 / 2 R_{\text {opt }}$ and $R_{\text {opt }}$ is the optimized ROC of the thermal lens. To get the optimal value of $A_{\text {opt }}$, the overlap integral $I$ between $E_{2}$ and $E_{3}$ is evaluated as

$$
\begin{aligned}
I\left(A_{o p t}\right)= & \int_{-\infty}^{\infty} E_{2}(x, z) \times E_{3} *(x, z) \mathrm{d} x \\
= & \left(\frac{2}{\pi}\right)^{1 / 2} \frac{1}{w(z)} \int_{-\infty}^{\infty} \exp -x^{2}\left[\frac{2}{w^{2}(z)}+i \frac{\pi}{\lambda} \frac{4 s(x)}{x^{2}}\right. \\
& \left.-i \frac{\pi}{\lambda} 4 A_{o p t}\right] \mathrm{d} x .
\end{aligned}
$$

The overlap integral of Eq. (15) represents the 1D amplitude coupling from the thermally aberrated reflected beam in the case of Fig. 4(a) and transmitted beam in the case of Fig. 4(b) into the fundamental Gaussian mode because $E_{3}$ has the form of a pure fundamental Gaussian mode. This perfect mode occurs because the thermal lens has been represented by a perfect spherical lens. The optimal value of $A_{o p t}$ is obtained by maximizing the overlap integral $I ; A_{o p t}$ will yield the optimal spherical approximation to the thermal lensing effect. The above integral can be written as

$$
\begin{aligned}
I(A)= & \left(\frac{2}{\pi}\right)^{1 / 2} \frac{1}{w(z)} \int_{-\infty}^{\infty} \exp -x^{2}\left[\frac{2}{w^{2}(z)}\right] \\
& \times \exp i\left[\frac{4 \pi}{\lambda_{1}} s(x)-\frac{\pi}{\lambda} 4 A_{o p t} x^{2}\right] \mathrm{d} x .
\end{aligned}
$$

Next, using Euler's identity, $e^{i \theta}=\cos \theta+i \sin \theta$ and noting that the integral $I$ is an even function of $x$, we write Eq. (16) as 


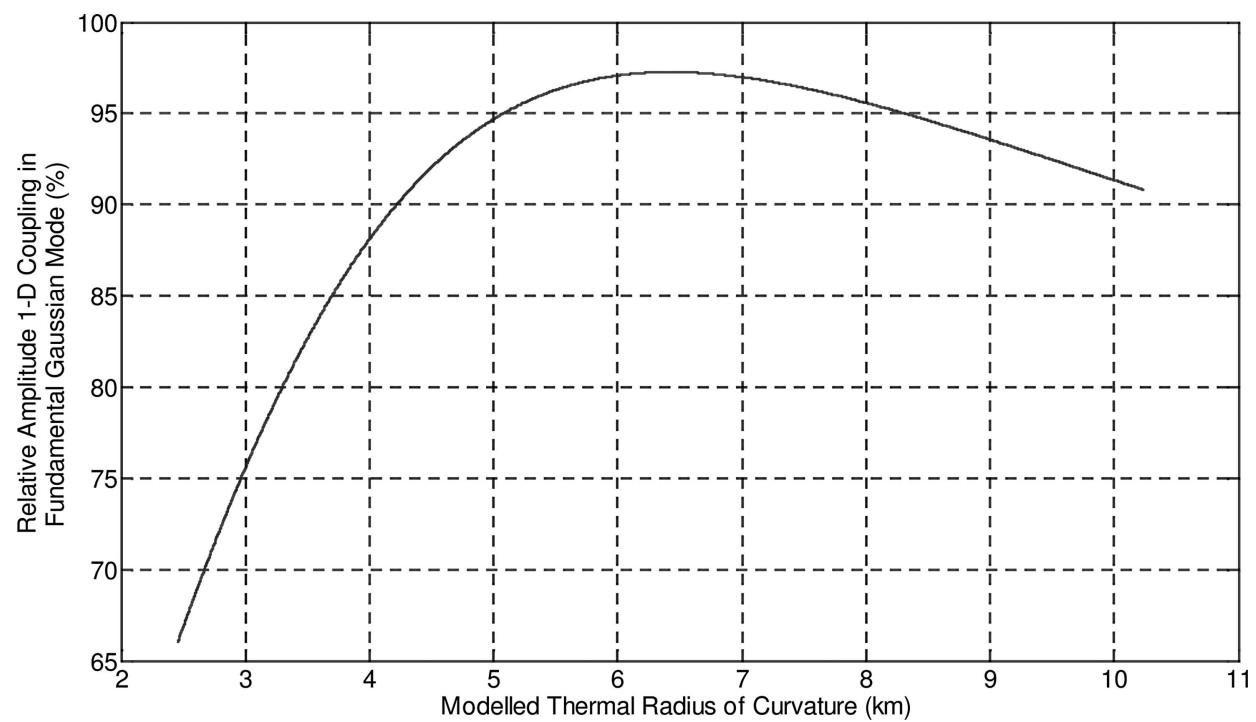

Fig. 5. The amplitude 1D coupling coefficient [Eq. (17)] for the case of substrate thermal lensing in LIGO test mass. The maximum occurs at a thermal ROC of $6.4 \mathrm{~km}$ indicating the optimal ROC associated with the thermal aberrations.

$$
\begin{aligned}
I(A)= & 2\left(\frac{2}{\pi}\right)^{1 / 2} \frac{1}{w(z)} \int_{0}^{\infty} \exp -x^{2}\left[\frac{2}{w^{2}(z)}\right]\left[\operatorname { c o s } \left\{\frac{4 \pi}{\lambda}[s(x)\right.\right. \\
& \left.\left.\left.-A_{o p t} x^{2}\right]\right\}+i \sin \left\{\frac{4 \pi}{\lambda}\left[s(x)-A_{o p t} x^{2}\right]\right\}\right] \mathrm{d} x .
\end{aligned}
$$

This integral can be evaluated numerically and the maximum of the integral is used to determine the optimal value of the ROC associated with the thermal lensing. This is applicable to both the thermal lensing in substrate and on the surface.

Continuing with the example of the advanced LIGO input test mass mirror, with the thermal aberrations shown in Fig. 3, the overlap integral and corresponding mode mismatch are shown in Fig. 5. The maximum of this overlap integral occurs at a thermal ROC of 6.4 $\mathrm{km}$. The optimal quadratic solution is plotted in Fig. 3 as a dotted-dashed curve. The optimal value of the integral also gives the maximum value of the $1 \mathrm{D}$ amplitude coupling coefficient; subtracting that amount from unity gives the 1D amplitude losses suffered by mode matching into an advanced LIGO optical cavity. The absolute losses increase as the optimal thermal lensing increases with increase in coating or bulk absorption, since as the thermal lensing increases, the difference between a quadratic profile and the actual lens profile created by the heating beam becomes larger and larger. This difference produces higherorder losses when the beam is either reflected or transmitted through such optics.

It is inevitable that when a high-power beam is reflected or transmitted by a mirror that is part of some Fabry-Perot cavity, a portion of the energy is deposited as heat in the coatings, giving rise to a physical deformation at the surface that changes the ROC of that element and thus changing the fundamental mode of that cavity. ${ }^{2,3,13}$ If the input beam is properly mode matched to the cavity at low powers, it becomes progressively more poorly matched at higher powers. To keep the coupling into this cavity maximal one can introduce an adaptive mode-matching telescope to control the beam size and waist location. For proper operation, a tunable optical element is required that can provide both positive and negative lensing elements. Such a system can be developed by using the technique proposed and demonstrated here, i.e., by controlled heating of elements with specific optical and physical properties.

\section{Application to Adaptive Optical Elements}

The phenomenon of thermal lensing can be used advantageously for creating adaptive optical elements. This can be done by either direct or indirect compensation. In direct compensation, an auxiliary heating beam is applied to the same optical element that produces the thermal distortion. In the case of indirect compensation, the heating beam is applied to a compensation element inserted near the thermally aberrated element to correct the thermal distortions. Control of the thermally induced optical profile can be realized by manipulating any of the following: (i) the intensity of the heating beam, (ii) the beam shape of the heating beam, (iii) the wavelength of the heating beam, or (iv) the properties of the compensation optics.

Typically, the intensity and the beam shape of the heating beam are the most easily adjustable adaptive control knobs. Nonetheless, the heating beam wavelength and the properties of the optical element can also greatly aid in designing an effective compensation scheme; of course, their adaptive control may be limited for practical purposes. Proper selection of these two factors can greatly reduce the requirements on the intensity and beam shape of the elements. As the optical properties (absorption) of the material depend upon the wavelength, changing the wavelength provides another means of control for a given material. For example, changing the heating 
beam wavelength to a more absorptive wavelength requires less heating or smaller thickness of the adaptive optical element for a given performance requirement.

A truly adaptive system will require both tunable positive and negative lenses. A positive lens has a quadratically decreasing radial optical path length whereas a negative lens has a quadratically increasing radial optical path length. As discussed above, heating of an optical element produces primarily two effects, namely, surface deformation and substrate refractive index variation. Based upon the beam shape, the material characteristics of that element, and the optical element orientation, the combination of these factors can produce either a positive or a negative lensing element. A figure of merit (FOM), based upon the material properties of the element is

$$
\begin{aligned}
\mathrm{FOM}_{M}= & {\left[\frac{\mathrm{d} n}{\mathrm{~d} T}+\alpha_{T}(1+v) \times(n-1)-\alpha_{T} \frac{n^{3}}{4} \frac{(1+v)}{(1-v)}\right.} \\
& \left.\times\left(p_{11}+p_{12}\right)\right] .
\end{aligned}
$$

Here $p_{11}$ and $p_{12}$ are the thermoelastic coefficients of the optical material specific for the [001] direction. ${ }^{14}$ $\mathrm{FOM}_{M}$ depends upon the signs and values of $\mathrm{d} n / \mathrm{d} T$, $\alpha_{T}$, and thermoelastic coefficients that may vary according to the geometry and orientation of the optical element. Here the spatial dependence of the thermal distortion is neglected in comparing the relative strengths of the thermal expansion and thermo-optic effects. If the value of $\mathrm{FOM}_{M}$ is positive (negative), the optical element will act as a positive (negative) lens when heated by a traditional Gaussian pump beam with a radially symmetric, monotonically decreasing intensity pattern. The situation can be reversed by applying an "inverse" Gaussian intensity pattern or in fact, in general, by heating with any pump beam whose intensity increases radially. However, this may require applying excessive heat as compared with the Gaussian beam case for the same amount of thermal lensing on account of the inward flow of heat in the element.2,13 Therefore, using a material with negative $\mathrm{FOM}_{M}$ is a better alternative to the radial heating.

There are a few potential disadvantages with such a system. The first are the higher-order losses owing to the deviation of the thermal lens profile from the optimal parabolic shape. Several solutions are available. A simple solution is to use a heating beam with a diameter larger than the probe beam. Then, aligning the probe beam perfectly around the optical axis of the heating beam ensures that the probe beam only sees the central portion of the thermal aberrations; these are very close to a quadratic profile. This method was successfully demonstrated in Ref. 4. However, increasing the pump beam diameter requires more power to create the same thermal lens. Another solution is to use a non-Gaussian shaped heating beam. For example, using a flat-top or "top-hat" beam as a heating beam will create a nearly ideal quadratic profile. Figure 6 shows the computed thermal lens created by

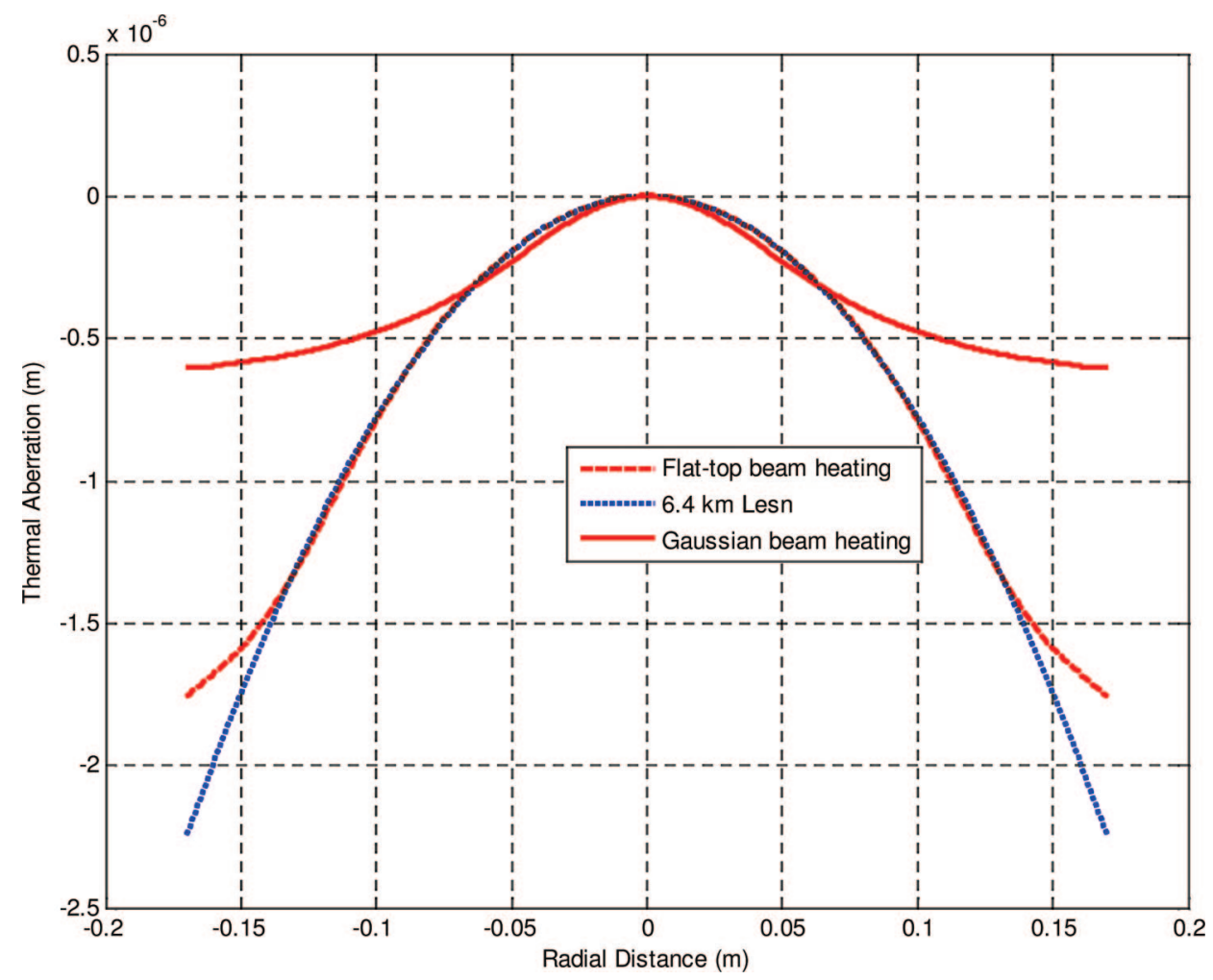

Fig. 6. (Color online) Thermal lens created through top-hat and Gaussian beam plotted using dashed curve and solid curve, respectively. The dotted line is an ideal thermal lens of $6.4 \mathrm{~km}$ focal length. 
Gaussian and a top-hat heating beams. The thermal lens created by the top-hat beam mimics an ideal lens for a much larger probe beam diameter; thus, the higher-order losses for top-hat beam are lower than for a Gaussian beam with the same probe beam diameter.

A second potential problem is the thermal depolarization associated with the thermoelastic effect. The complete theory of thermal depolarization is presented in Ref. 14. For materials such as fused silica (FS), with a very low thermal expansion coefficient, we calculate that the thermal depolarization is of the order of a few tens of parts per $10^{6}$. This effect becomes important for the negative thermo-optic coefficient materials, which usually have large thermoelastic coefficients. However, this effect can be reduced significantly by selecting appropriate orientations.

The third potential problem comes about from the specific choice of implementation geometry. The registry of the heating and probe beams on the compensation element can be performed either collinearly using dichroic beam combining optics or via irradiation by the heating and probe beams on the opposite sides of a reflective element. However, there may be situations where nonnormal incidence of the probe beam is unavoidable. In this case, the beam pointing will change as a function of pump beam power due to the induced index gradient. A first-order estimate of the beam deviation can be made by treating the thermal lens as a thick lens. A general $A B C D$ matrix of a thick lens is given by

$$
\left[\begin{array}{ll}
A & B \\
C & D
\end{array}\right]=\left[\begin{array}{cc}
1-(h / n)\left(P_{1}\right) & h / n \\
-\left(P_{1}+P_{1}-P_{1} P_{2} h / n\right) & 1-(h / n)\left(P_{1}\right)
\end{array}\right]
$$

where $P_{1,2}=(n-1) / R_{1,2}$ with $R_{1(2)}$ being the ROC of the front (back) surface of the lens. The displacement and angular deviation of the transmitted and reflected beam from the thermal lens can then be calculated by using $A B C D$ propagation laws. If $x_{1}\left(x_{2}\right)$ and $\theta_{1}\left(\theta_{2}\right)$ are the incident (final) beam displacement from the optical axis and the angle of incidence (refraction), respectively, then these quantities are related by

$$
\left[\begin{array}{c}
x_{2} \\
\tan \theta_{2}
\end{array}\right]=\left[\begin{array}{ll}
A & D \\
B & C
\end{array}\right]\left[\begin{array}{c}
x_{1} \\
\tan \theta_{1}
\end{array}\right]
$$

where the $A B C D$ matrix is given in Eq. (19). Note that this model is only a first-order estimate of the beam walking. More precise models can be developed by using beam propagation equations in inhomogeneous anisotropic medium.

Another important issue to consider is the time response of the system. The Hello and Vinet theory suggests a characteristic response time of $\tau_{c}=C \rho a^{2} / \kappa$, where $\tau_{c}$ is the characteristic time and $\rho$ is the density of the medium.5,6 This formula is applicable to the systems where the heating beam is the probe beam itself. In reality, the temperature evolution of the optical element varies with the radial and axial location. For example, the temperature of the center of the optical element increases faster than the off-axis locations. In our case, the heating is due to an auxiliary heating beam and the probe beam only samples a very small portion of the heated volume. Therefore a reduced diameter $\left(r_{e}\right)$ should be used to predict the response time. One way to determine this reduced radius is to measure the response time and infer the thermal coefficients from the results. However, intuitively, the location of the probe beam should be within $r_{c}$.

Furthermore, as the amount of probe beam displacement increases with increased heating-beam power, the response time should also increase. Here a parallel can be drawn with bolometer theory, where the response time also depends upon the external heat dissipation. ${ }^{15}$ Therefore, the response time $\tau_{P B D}$ can be defined as

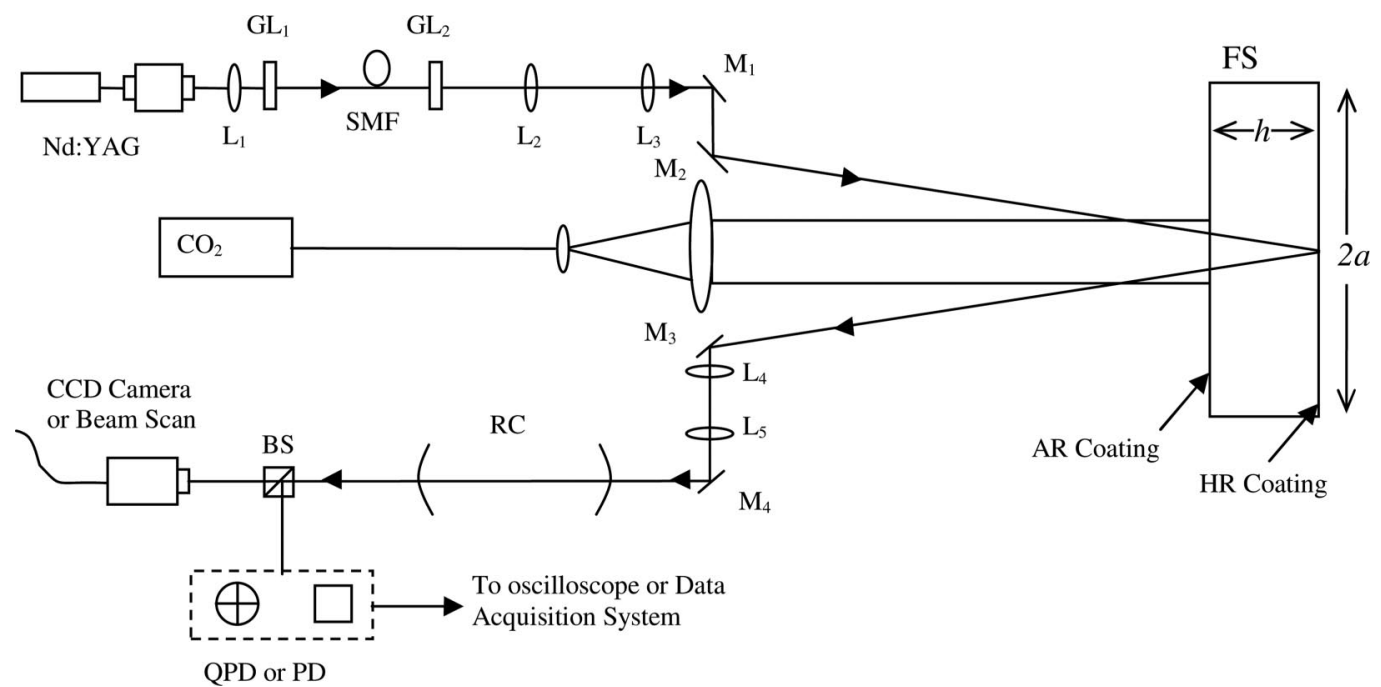

Fig. 7. Experimental arrangement of the adaptive optical system. SMF, single-mode fiber; L, lens; M, mirror; PD, photodetector; QPD, quad photodetector; GL, graded index lens; BS, beam splitter; and RC, scanning Fabry-Perot analyzer cavity. 


$$
\tau_{P B D}=\frac{C \rho r_{e}^{2}}{\kappa-\beta P} .
$$

Here $\beta$ is a parameter that represents the effect of auxiliary beam power absorption in units of $\mathrm{K}^{-1} \mathrm{~m}^{-1}$. For small values of $P$, Eq. (22) can be approximated as

$$
\tau_{P B D}=\frac{C \rho r_{e}^{2}}{\kappa}-\frac{C \rho r_{c}^{2}}{\kappa^{2}} P=\frac{C \rho r_{e}^{2}}{\kappa}+\beta_{r} P .
$$

Here $r_{e}$ and $\beta_{r}$ can be determined by curve fitting as we show in Section 4.

\section{Experimental Demonstration}

The experimental architecture is shown in Fig. 7. A 5 $\mathrm{cm}$ diameter $0.95 \mathrm{~cm}$ thick UV-grade FS mirror is used as the adaptive compensation element. The FS mirror is mounted in a holder such that it rests on three Teflon contacts. Hence negligible heat flows through conductance. Although FS is in air but in the limit of low temperature rise, the dominant heat escape mechanism is radiation. Relevant material parameters for FS are shown in Table 1. For the probe beam, a $1.064 \mu \mathrm{m}$ single longitudinal mode Nd:YAG laser is used. The transverse mode of the probe beam is "cleaned" using a single-mode fiber. A pair of lenses is used to expand the beam. The beam diameter of the probe beam at the front face of the FS mirror is $1.8 \mathrm{~mm}$. The test mirror is oriented such that the high-reflectance surface of the mirror is at the rear side with regard to the incident probe beam, allowing us to take advantage of the integrated thermal optical path deformation in the substrate in a doublepass geometry. The probe beam hits the test mirror at an angle of $\theta=3.0^{\circ}$ with the normal. After reflecting from the mirror, the beam is focused by a $75 \mathrm{~cm}$ lens onto a pyroelectric beam scanner. ${ }^{16}$ The Gaussian parameters are measured at various positions in the optical system to track the beam waist and location throughout the system.

A $10 \mathrm{~W} \mathrm{CO}_{2}$ gas laser with a wavelength of $10.6 \mu \mathrm{m}$ and a Gaussian profile is used as a heating beam. The FS mirror substrate is essentially transparent for the $1.064 \mu \mathrm{m}$ wavelength probe beam, while the penetration length at $10.6 \mu \mathrm{m}$ is approximately $56 \mu \mathrm{m}$. Thus, all the energy in the heating beam is absorbed very near to the surface. The heating beam is expanded to $2.0 \mathrm{~cm}$ with a telephoto lens system, providing a heating beam to probe beam diameter ratio of more than 10, minimizing induced higher-order losses in the probe beam.

The test plate was heated with the $\mathrm{CO}_{2}$ laser running at various power levels, and a beam scan was performed on the probe beam after it had been reflected from the mirror and passed through a focusing lens. Two sets of data were taken; one at $[0,1,2,3] \mathrm{W}$ and a second at $[0,0.5,1.5,2.5] \mathrm{W}$ heating-beam power. The two different data sets correspond to two slightly different beam scanning positions. The probe beam power is approximately $100 \mathrm{~mW}$ and has a negligible effect on the thermal lensing in the test mirror. The beam divergence data are shown in Fig. 8 for the

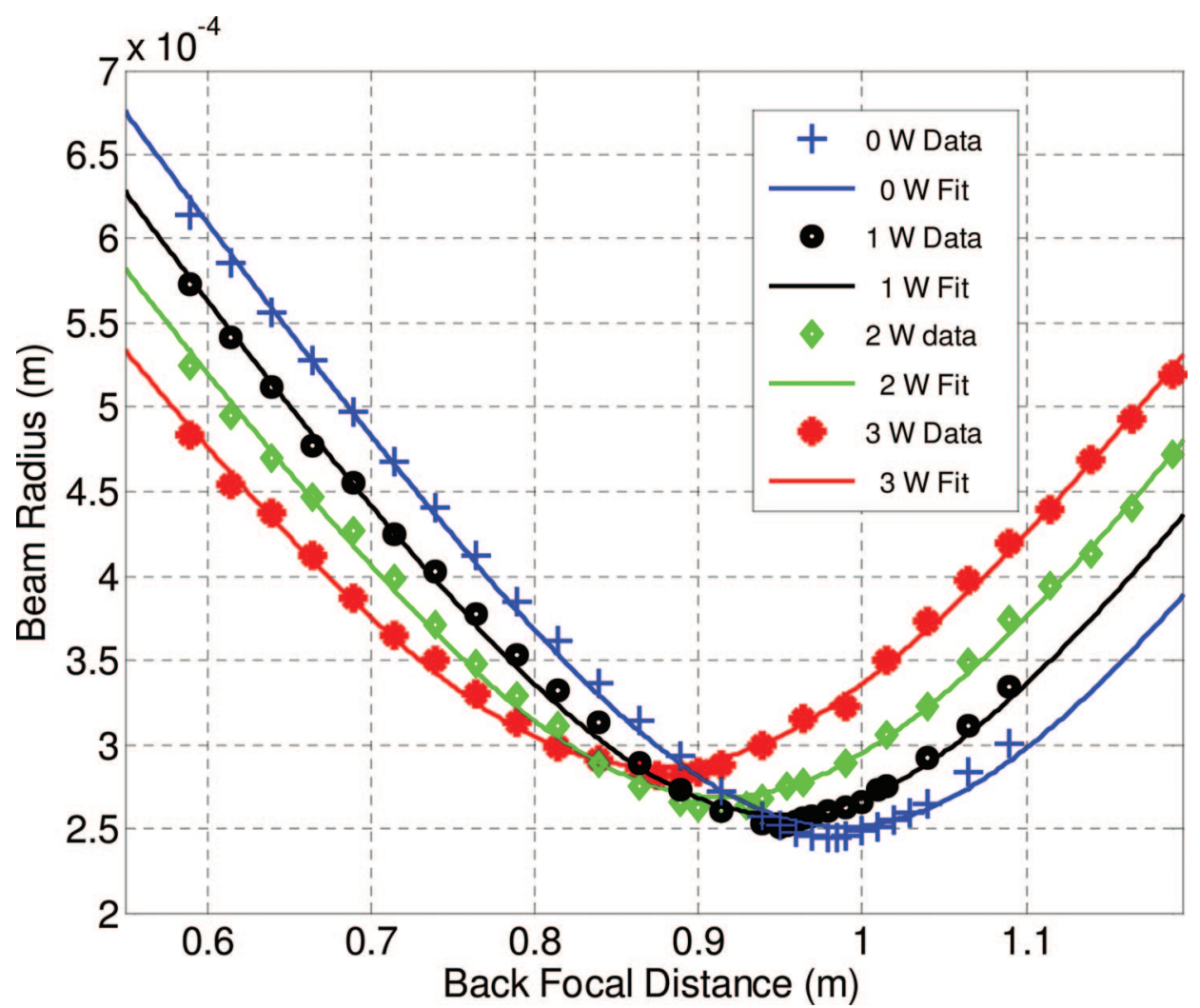

Fig. 8. (Color online) Beam profile data and the corresponding Gaussian fit at $[0,1,2,3] \mathrm{W}$ absorbed power. 


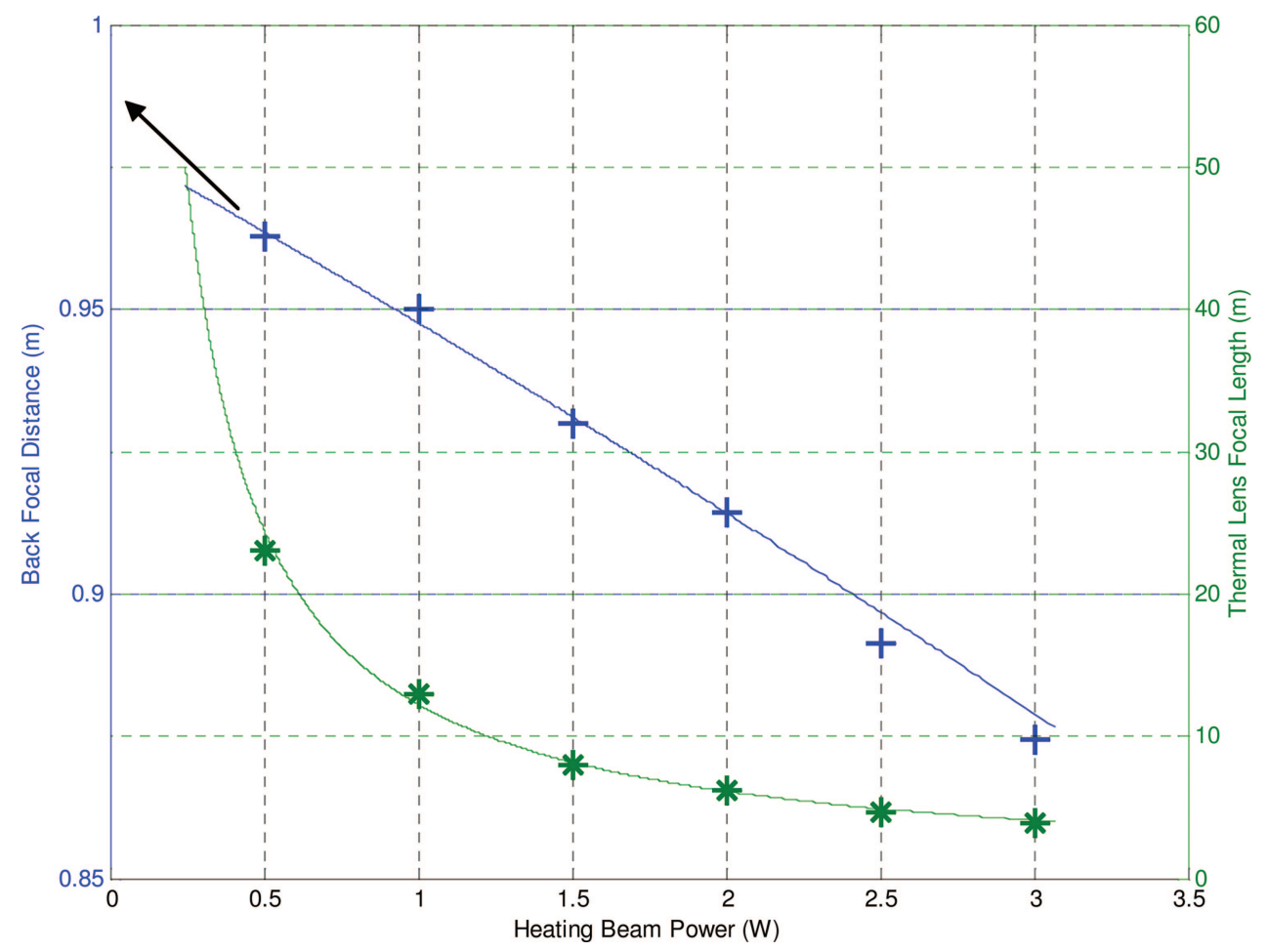

Fig. 9. (Color online) Calculated and measured back focal distance as a function of absorbed power (left axis) and the corresponding focal length of the thermal lens (right axis) as a function of the absorbed power. The measured data are shown as symbols.

first data set. The data points at various power levels are represented by markers while a Gaussian beam fit is shown as solid curve. The back focal length, i.e., the distance from the focusing lens to the minimum beam waist position, is determined by the Gaussian fit parameters. Using this back focal length data and $A B C D$ matrices, the effective focal length of the test plate is calculated. Figure 9 shows the experimentally determined back focal length plotted on the left axis and the resultant focal length of the test plate plotted on the right $y$ axis as a function of pump beam power.

The theoretical model presented earlier is used to compute the focal length of the test plate at various power levels. It is important to note that there are no adjustable parameters; only the known optical and physical parameters are used. The theoretical value of the lens power (in diopter or $\mathrm{m}^{-1}$ ) as a function of heating beam power is shown in Fig. 10 along with the experimentally measured data. The two values are in excellent agreement. The slope of the curve gives a value of 0.082 diopter $/ \mathrm{W}$ of pump beam.

The loss of power in higher-order modes can be calculated theoretically by evaluating the overlap integral defined in Eq. (17) and subtracting it from unity. The theoretically predicted power loss is shown in Fig. 11 as a function of absorbed power (left axis). The modeled ROC is also shown (right axis). Note that the calculated decrease in power at $5 \mathrm{~W}$ is only $0.015 \%$, close to the measurement noise. Hence, it can be assumed that the system has no appreciable higher-order mode content.
Experimentally, this was verified by using a FabryPerot cavity to analyze both the tilt and higher-order mode content. ${ }^{17}$ Thermally induced beam pointing is inferred from the loss in the fundamental mode and the power transferred to higher-order $\mathrm{TEM}_{10,01}$ modes of an initially mode-matched cavity. We measured this using a combination of a photodiode and beam profiling of the transmitted or reflected mode. The alignment was optimized such that only the $\mathrm{TEM}_{00}$ mode is resonant in the test cavity at zero heatingbeam power. As the test mirror was heated by the pump beam, higher-order tilt modes are induced due to the changes in the propagation and angle and displacement of incoming probe beam with respect to the cavity axis while higher-order Laguerre Gauss ${ }^{16}$ (mainly bull's-eye) modes is introduced due to change in waist size and location of the probe beam inside the cavity. The coupling to the cavity is then reoptimized by changing the tilt and the location of the cavity for different pump beam powers. At all heating-beam power levels, we find that virtually the same amount of probe-beam power is stored in the cavity. The negligible decrease in the resonant $\mathrm{TEM}_{00}$ mode after increasing the pump-beam power and realigning the cavity, indicates that no appreciable higher-order modes (beyond tilt modes induced by beam steering and bull's-eye mode caused by wavefront curvature changes) are produced by the adaptive lens over the range of experimental parameters explored.

We also attempted to measure the beam deflection angle with a quadrant photodetector located $1 \mathrm{~m}$ from the compensation mirror. At even the highest heat- 


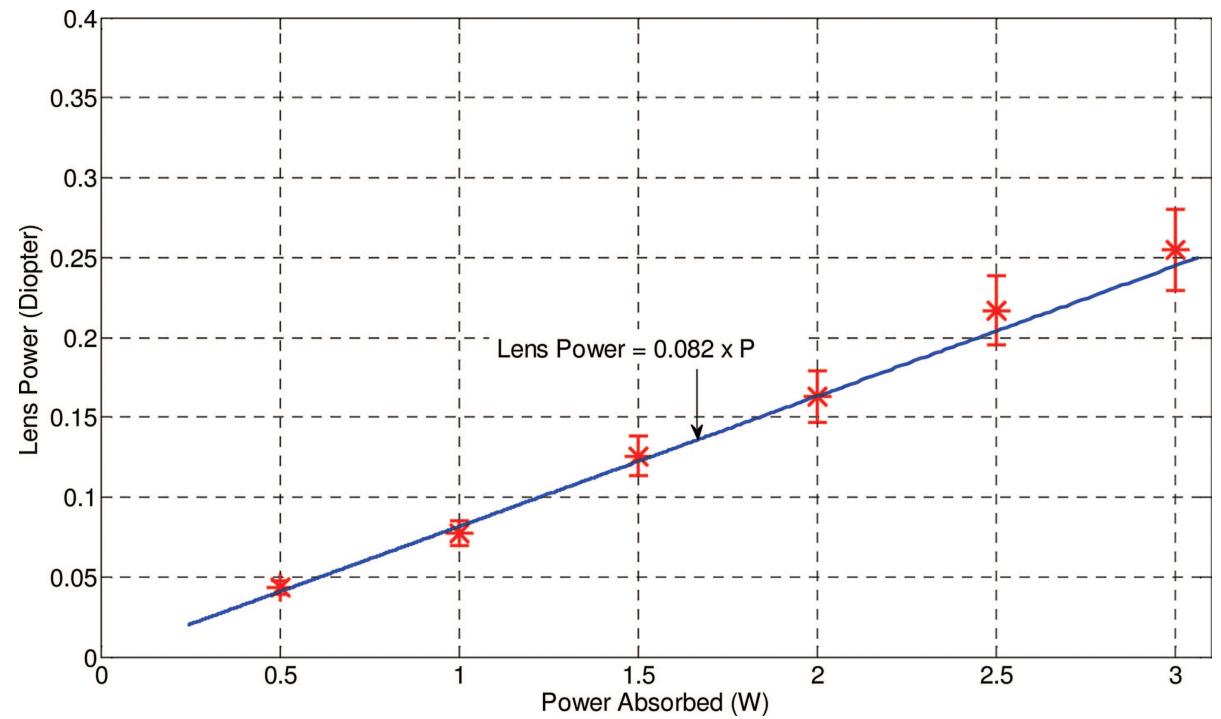

Fig. 10. (Color online) Measured values of lens power as a function of absorbed power is shown as symbols, with error bars. A linear fit to the data gives a slope of 0.082 diopter/W.

ing power, the beam deflection was experimentally unresolved $(<0.15 \mathrm{mrad} / \mathrm{W})$, so the angular beam deviations were estimated by approximating the adaptive lens as a thick lens using Eq. (20). Numerically, an angular shift of $0.028 \mathrm{mrad} / \mathrm{W}$ was determined. To enhance the amount of induced beam steering, an incident angle of $18.5^{\circ}$ was used, where a substantial amount of tilt is expected. The probe beam is first centered on the quad photodetector (QPD) and the reading on the attached micrometer is noted. Next the auxiliary heating beam is turned on, heating the test mirror. As a result, the probe beam suffers a displacement and the reading on the QPD is changed. The micrometer is then used to recenter the probe beam on the QPD. The change in micrometer reading gives the direct measurement of probe-beam displacement. To confirm this mea- surement, the heating beam is turned off and the probe beam was observed to move in the opposite direction as the test plate cooled to room temperature. Again, the probe beam moved from the center position on the QPD and the micrometer was used to recenter the QPD. An average of these two readings was used as the beam displacement caused by thermal lensing of the auxiliary heating beam. These data are shown in Fig. 12, and agree well with the predicted results using the thick lens approximation. Curve fitting to Fig. 12 provides a beam deviation of $0.16 \mathrm{mrad} / \mathrm{W}$. The cavity analyzer was also used to measure the amount of power in higher-order modes. At each power level, the cavity was reoptimized by changing the $x$-axis and $y$-axis tilt of the beam through mirrors to minimize the induced thermal steering and shifting the cavity along the

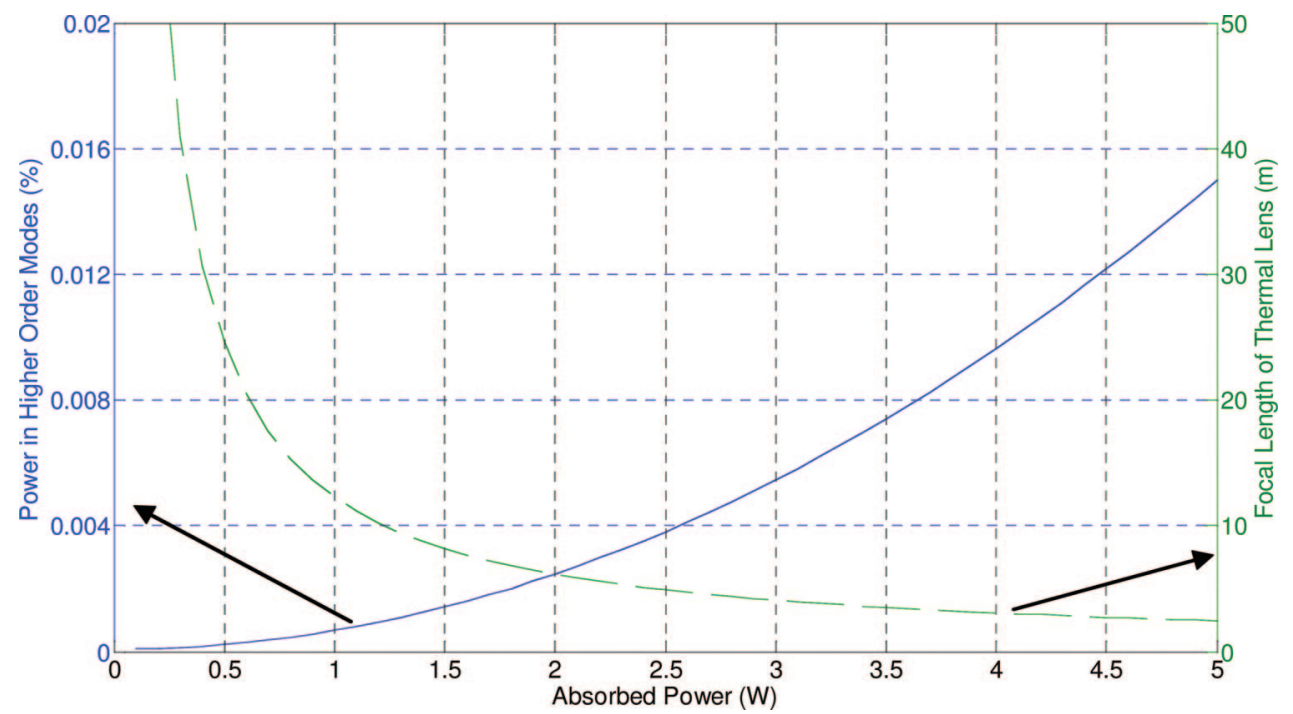

Fig. 11. (Color online) Prediction of higher-order losses in the experimental setup of Fig. 7 (left axis) and the corresponding focal length (right axis) as a function of absorbed power. 


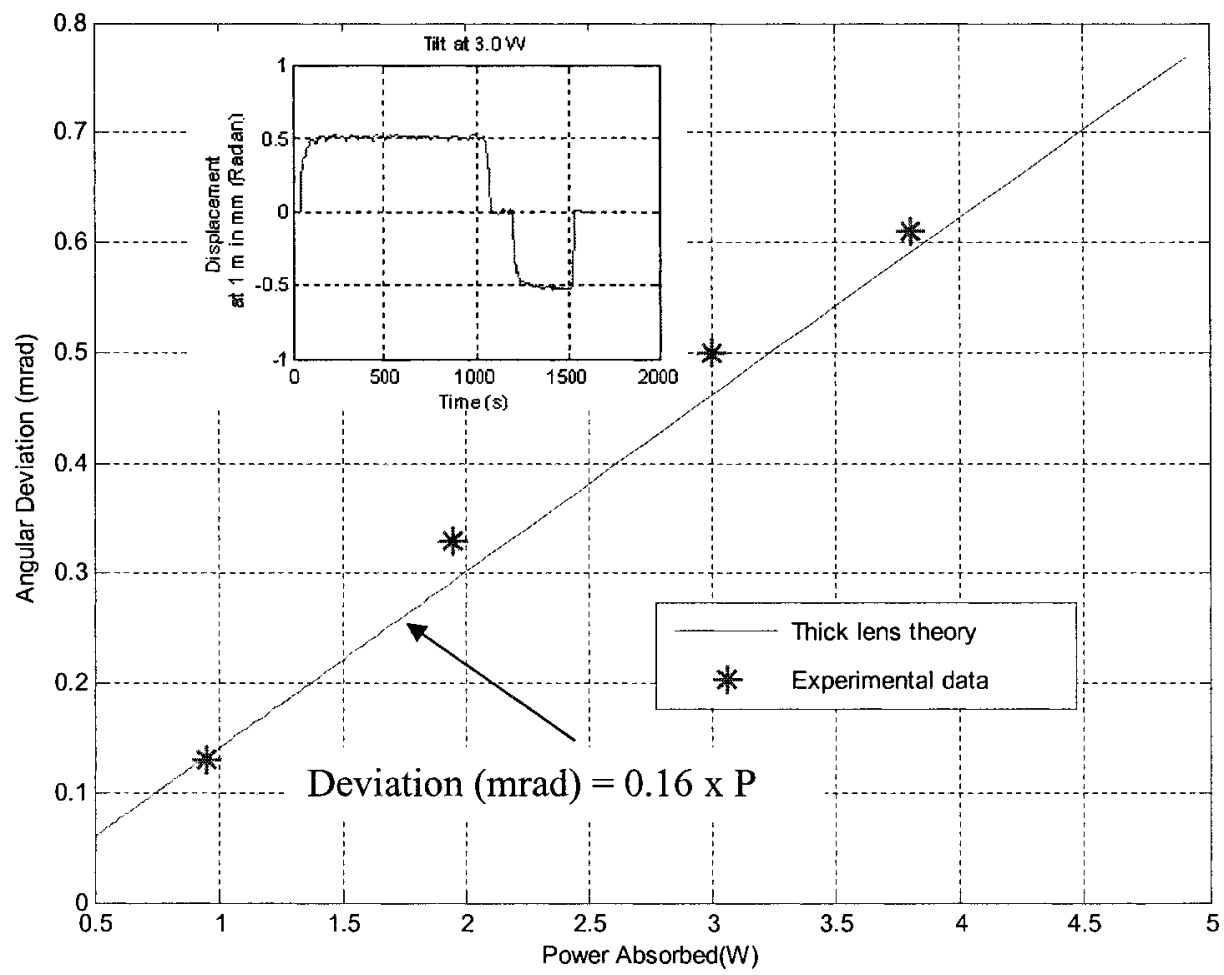

Fig. 12. Deviation of the probe beam from its base position at room temperature as a function of absorbed power. The solid line is the theoretical prediction using thick lens approximation. The slope gives $0.16 \mathrm{mrad} / \mathrm{W}$ deviation at $18.5^{\circ}$ incidence angle of the probe beam.

axial direction and/or changing the position of the focusing lenses to remode-match the beam. More than $99 \%$ of the power can be maintained in the resonant $\mathrm{TEM}_{00}$ mode by tilt and position control, showing that there is no appreciable higher-order mode content beyond tilt.

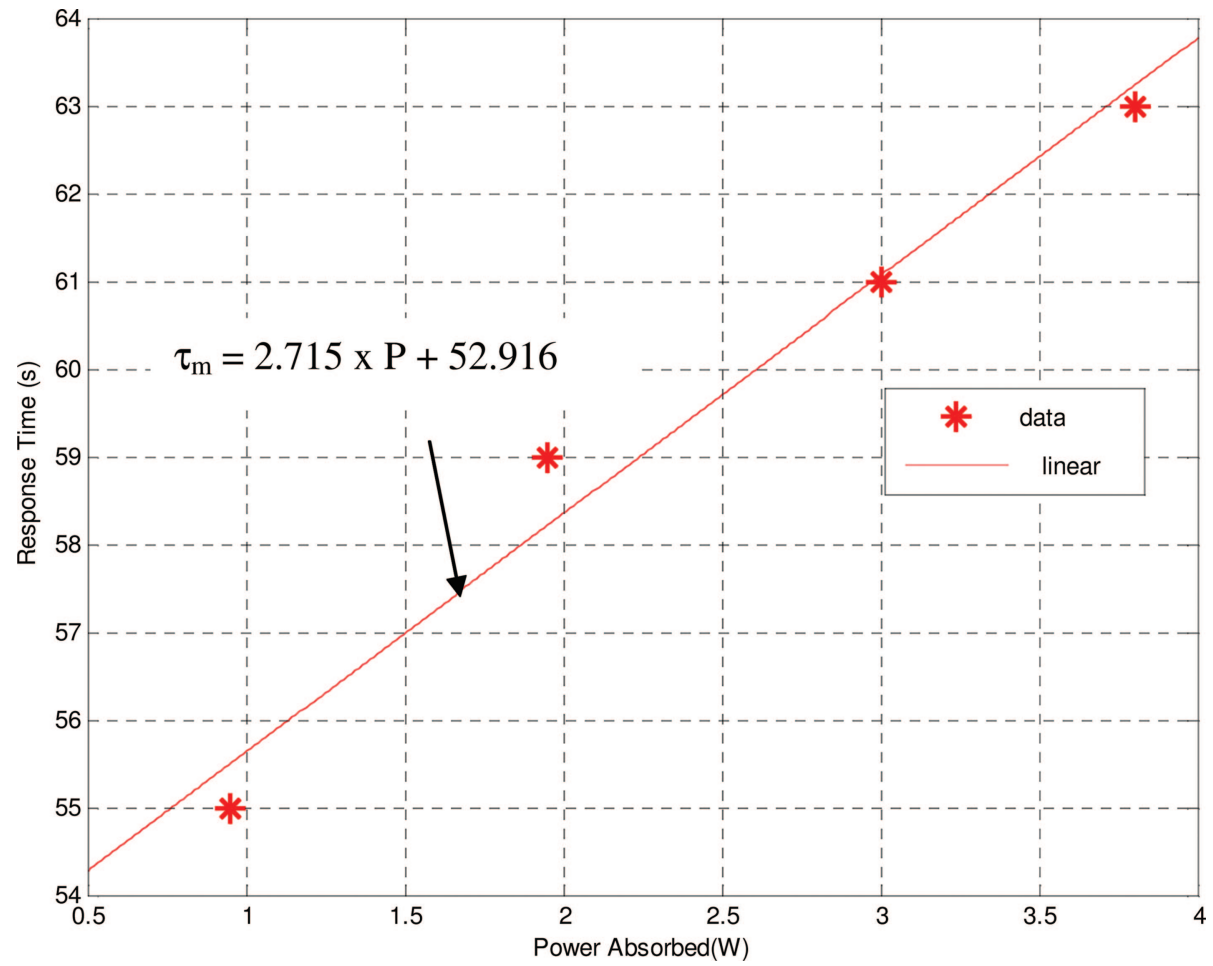

Fig. 13. (Color online) Response time of the demonstrated system as a function of absorbed power. The slope is $2.715 \mathrm{~s} / \mathrm{W}$ over an essential characteristic time delay of $52.916 \mathrm{~s}$. 
The inset in Fig. 12 shows data as a time series and thus gives valuable insight into the thermal constants or the response time of the system. The measured response time $\tau_{m}$ defined as the time required to reach $95 \%$ of the final value is plotted in Fig. 13. A linear curve fit provides a value of $\tau_{m}=2.715 P$ +52.916 where $P$ is the power of the auxiliary beam. Using the material properties in Table 1 and comparing the curve fit equation with Eq. (22) gives a reduced radius of $2.1 \mathrm{~mm}$, slightly larger than the offset of the probe beam from the center of the optical element. This reduced radius is somewhat intuitively expected. Thus the response time has a base value of $53 \mathrm{~s}$ and $\sim 2.7 \mathrm{~s}$ additional delay per Watt of the heating beam power absorbed in this experiment. Of course, these response times are specific to our particular choices of experimental parameters and will change for differing values for the probe radius and the size of the adaptive compensation plate.

\section{Conclusion}

In conclusion, an adaptive system for control of firstorder laser modal properties is presented that can be used to change dynamically the wavefront radius of curvature of an optical beam. Heating of the optical element can be used to create a positive or negative lensing element based upon the shape and the material properties of the adaptive optical element. A detailed model for the thermal lensing is presented. A new technique based upon the overlap integral is presented for correct ROC estimation. A simple model for beam displacement is presented. The thermal response time has also been measured and found to be close to a minute. Experimental results agree well with theory. Applications include adaptive beam coupling into high-power laser systems and thermal lensing compensation.

The authors gratefully acknowledge the support of the National Science Foundation through grants PHY-0555453 and PHY-0354999. We also thank the LIGO Science Collaboration internal review for a careful review of our manuscript.

\section{References}

1. J. D. Mansell, J. Hennawi, E. K. Gustafson, M. M. Fejer, R. L. Byer, D. Clubley, S. Yoshida, and D. Reitze, "Evaluating the effect of transmissive optic thermal lensing on laser beam quality with a Shack-Hartmann wavefront sensor," Appl. Opt. 40, 366-368 (2001).

2. R. Lawrence, M. Zucker, P. Fritschel, P. Marfuta, and D. Shoemaker, "Adaptive thermal compensation of test masses in advanced LIGO," Class. Quantum Grav. 19, 1803-1812 (2002).

3. J. Degallaix, C. Zhao, L. Ju, and D. Blair, "Thermal lensing compensation for AIGO high optical power test facility," Class. Quantum Grav. 21, S903-S908 (2004).

4. V. Quetschke, J. Gleason, M. Rakhmanov, J. Lee, L. Zhang, K. Yoshiki Franzen, C. Leidel, G. Mueller, R. Amin, D. B. Tanner, and D. H. Reitze, "Adaptive control of laser modal properties," Opt. Lett. 31, 217-219 (2006).

5. P. Hello and J. Vinet, "Analytical models of thermal aberrations in massive mirrors heated by high power laser beams," J. Phys. (France) 51, 1267-1282 (1990).

6. P. Hello and J. Vinet, "Analytical models of transient thermoelastic deformations of mirrors heated by high power CW laser beams," J. Phys. (France) 51, 2243-2261 (1990).

7. J. D. Foster and L. M. Osterink, "Thermal effects in Nd:YAG laser," Appl. Opt. 41, 3656-3663 (1970).

8. C. E. Greninger, "Thermally induced wavefront distortions in laser windows," Appl. Opt. 41, 549-552 (1986).

9. K. A. Strain, K. Danzmann, J. Mizuno, P. G. Nelson, A. Rüdiger, R. Schilling, and W. Winkler, "Thermal lensing in recycling interferometric gravitational wave detectors," Phys. Lett. A 194, 124-132 (1994).

10. W. Winkler, K. Danzmann, A. Rüdiger, and R. Schilling, "Heating by optical absorption and the performance of interferometric gravitational-wave detectors," Phys. Rev. A 44, 7022-7036 (1991).

11. R. G. Beausoleil, E. K. Gustafson, M. M. Fejer, E. D’Ambrosio, W. Kells, and J. Camp, "Model of thermal wave-front distortion in interferometric gravitational-wave detectors. I. Thermal focusing,” J. Opt. Soc. Am. B 20, 1247-1268 (2003).

12. A. Weinstein, "Advanced LIGO optical configuration and prototyping effort," Class. Quantum Grav. 19, 1575-1584 (2002).

13. R. Lawrence, D. Ottaway, M. Zucker, and P. Fritschel, "Active correction of thermal lensing through external radiative thermal actuation," Opt. Lett. 29, 2635-2637 (2004).

14. E. Khazanov, N. F. Andreev, A. Mal'shakov, O. Palashov, A. K. Poteomkin, A. Sergeev, A. Shaykin, V. Zelenogorsky, I. A. Ivanov, R. Amin, G. Mueller, D. B. Tanner, and D. H. Reitze, "Compensation of thermally induced modal distortions in Faraday isolators," IEEE J. Quantum Electron. 40, 1500-1510 (2004).

15. F. J. Low, “Low-temperature geranium bolometer,” J. Opt. Soc. Am. 51, 1300-1304 (1961).

16. Beam Scan, Model XYFIR, Photon Inc., http://www.photoninc.com.

17. D. Z. Anderson, "Alignment of resonant optical cavities," Appl. Opt. 23, 2944-2949 (1984). 